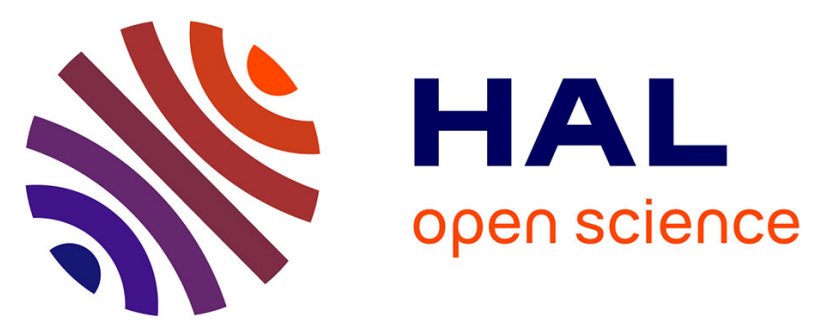

\title{
Insights into the Trilateral Relationship of Crowdfunding Campaigns, Open Source and Communities
}

\author{
Patricija Ilin, Dimitrios Platis, Imed Hammouda
}

\section{To cite this version:}

Patricija Ilin, Dimitrios Platis, Imed Hammouda. Insights into the Trilateral Relationship of Crowdfunding Campaigns, Open Source and Communities. 14th IFIP International Conference on Open Source Systems (OSS), Jun 2018, Athens, Greece. pp.26-36, 10.1007/978-3-319-92375-8_3 . hal01875485

\author{
HAL Id: hal-01875485 \\ https://hal.inria.fr/hal-01875485
}

Submitted on 17 Sep 2018

HAL is a multi-disciplinary open access archive for the deposit and dissemination of scientific research documents, whether they are published or not. The documents may come from teaching and research institutions in France or abroad, or from public or private research centers.
L'archive ouverte pluridisciplinaire HAL, est destinée au dépôt et à la diffusion de documents scientifiques de niveau recherche, publiés ou non, émanant des établissements d'enseignement et de recherche français ou étrangers, des laboratoires publics ou privés. 


\title{
Insights into the trilateral relationship of crowdfunding campaigns, open source and communities
}

\author{
Patricija Ilin ${ }^{1}$, Dimitrios Platis ${ }^{2}$ and Imed Hammouda ${ }^{3}$ \\ 1 Univerity of Gothenburg, Sweden \\ ilinpatricia@outlook.com \\ 2 Aptiv PLC \\ dimitrios.platis@aptiv.com \\ 3 Mediterranean Institute of Technology, South Mediterranean University, Tunisia \\ Chalmers and University of Gothenburg, Sweden \\ imed.hammouda@medtech.tn
}

\begin{abstract}
Crowdfunding campaigns enable individuals to bring their ideas to production by appealing directly to the end-market and the global community. A number of these projects are open source, seemingly, counteracting the funding process. We interviewed founders, developers and managers of 13 crowdfunding initiatives involving open source products to determine how communities, crowdfunding campaigns and open source are associated. Our findings verified the existence of common characteristics among the cases, the emergence of a family-like relationship between the organizers and the community, as well as the community perceived as a success factor. We suggest that the development of certain niche products inherently leads to the adoption of open source as a licensing model and crowdfunding as the capital gathering process.
\end{abstract}

\section{INTRODUCTION}

During the recent years, we have seen the rise of start-up companies as well as their contribution to the economy by creating job positions, enabling the youths to innovate and taking their ideas to the market. One way these ideas get investment to reach the market and influence market trends is via crowdfunding platforms with crowd raised contributions, such as Kickstarter, Indiegogo and Crowd Supply. According to Massolution's crowdfunding report, summed up in [2], the total global crowdfunding revenue was over $\$ 16.2$ billion worldwide in 2014. The report further shows that the leading regions were N. America, Asia and Europe, and the crowdfunding models that had grown the most in percentage were the donation and the equity-based models.

Lately, we have witnessed the merge of these two trends, open source and crowdfunding, often ending up in successful projects. What ties the open source and crowdfunding together is the idea of social influence from a devoted crowd movement that supports the organizers and steers the outcome of a project. 
Social networking is, in this case, a fundamental component for both of the cases. Because of the networking attribute, the crowdfunding backers who support a project, typically help invest in it and market it through word of mouth among their friends and families on websites such as Facebook and Twitter. Likewise in open source, communities can be formed that lead to companionship and inspire the individuals to not only contribute to a project but also engage into activities to popularize it, as in the case of Linux User Groups [1].

Previous research in this area is mainly focused on what motivations people have for investing in a crowdfunding project (e.g. [3], 4]) or what motivations they have for participating in an open source community (e.g. [5, 6] and [7]). Other research also sheds a light on what impact social media has on crowdfunding projects (e.g. [8]) or what role communities in general play in crowdfunding projects (e.g. 9] and [10]).

Despite many open source products being crowdfunded during the latest years, there has not been enough research to shed light on the characteristics of those campaigns and how they are associated with the corresponding communities. This study will aim to fill this gap, by attempting to determine the characteristics the specific crowdfunding campaigns, the communication process between the campaign organizers and the communities as well as the possible impact of the communities when crowdfunding an open source project.

\section{BACKGROUND}

\subsection{Crowdfunding}

Obtaining resources for a start-up or a product is often considered challenging. Typically, it can require a loan, finding investors, applying for a grant etc. When these tactics are not fruitful or sufficient, online crowdfunding can come in handy. Crowdfunding is an alternative way to raise money with the help of a large number of individuals from all over the world, who see potential in a product, project, social cause or service. The project can be anything from a physical product such as a smartphone case to a trip, book publishing, the start-up of a business, a concert or an expense (e.g. medical). The crowdfunding methods are typically based on donations, peer-to-peer or peer-to-business loans, rewards or equity, meaning that people who fund products can become co-owners.

In our research, we investigate initiatives launched on reward-based platforms, focused on physical products. They have adopted an all-or-nothing model, i.e. contributions will be returned unless the project reaches its funding goal.

\subsection{Communities}

Throughout the years, communities have been examined from different perspectives by researchers. Shared values and interests are what pervades the communities and results in mutual relationships and unselfish acts of kindness to one another. Findings by Li, Browne \& Wetherbe (2006) [12] as well as Wu, Chen \& 
Chung (2010) [13] show that the more values the members of a community share such as goals, appropriate behavior and policies, the stronger the competence, commitment and altruism is among the members. Additionally, the members' bond results in a higher level of satisfaction and enhances belongingness.

This view is further elaborated in [14] where it is claimed that apart from homogeneous behaviors, interpersonal relationships and bonds need be the foundation of the community for it to thrive. This leads to a knowledge community or network of practice, traditionally called a community of practice. These communities describe big groups or networks of people online, that may or may not know each other or meet face to face. They share common goals and purposes and use communication to fulfill these goals ([15], [16]).

\subsection{Doing business with open source}

One of the most crucial decisions when doing business with open source software is the license under which the derived works can be distributed. That being said, not all open source products have the same profit potential. Krishnamurthy (2003) divides OSS products into categories based on their importance and customer applicability [17. In this study, we investigated cases with products of lower customer applicability which, depending on their importance, can be regarded as high or low profile nichers.

Based on the nature of typical open source crowdfunding campaigns, we determined three business models as most applicable, taken from [18] and [19]. The Support seller model, which involves releasing the source while charging for services such as packaging, branding, distribution, customizing and supporting it. Brand licensing aims to sell the trademark. Finally, in Loss leader model, an open source product is used for marketing purposes to attract attention towards another, more profitable, product.

\subsection{Previous studies}

Gerber, Hui and Kou (2012) have been looking at the motivations for posting and funding crowdfunding projects online. Their study shows that the participationmotivations among crowdfunding project creators depend on the idea of strengthening commitment to community members and their feedback 3. Similarly, Brabham (2010:1139-1140) reveals that members are driven by opportunities in the form of money, skills and creating a portfolio. Additionally, he characterizes members "vibrant and obsessed" and deems these traits essential for a thriving crowdsourcing community.

Moreover, Hars and $\mathrm{Ou}$ (2001) have divided the motivation of members in an open source community in intrinsic e.g. altruism and identification with a community and extrinsic, direct compensation and personal needs [7. On the other hand, Lakhani and Wolf (2003), propose creativity as the main motive [6].

Regarding the impact of social media on crowdfunding projects, Lu, Xie, Kong and Lu (2014) mapped principles that have a positive effect on crowdfunding. They have observed that early promotional activities are strongly connected 
to the outcome and stress the benefit of using multiple platforms for promotion [8. As to the impact of communities, Bard, Brannström and Fahlberg (2014) highlight the importance of recognizing them as more than just financers. They are a devoted group sharing ideas and information [10. Finally, Matheus (2016) suggests the success rate of a crowdfunding platform depends on creators backing other projects, lessons learned and connection with the backers [9].

\section{RESEARCH QUESTIONS \& METHODOLOGY}

In the current work we investigate the socio-technical attributes present in crowdfunding campaigns of open source products, from the perspective of the campaign organizers. Utilizing the research questions as the protocol to help us explore the topic, we begin by identifying the advantages and disadvantages hereditary in such campaigns as well as the business aspects are relevant. Next, we track the communication tools and how they are used as well as investigate the relationship between the organizers and the community. Finally, we attempt to determine the impact of the communities by combining the results of the previous questions with empirical data.

- RQ1: What are the characteristics of a crowdfunding campaign of open source products?

- RQ2: How can the relationship and communication between the campaign organizers and the community be described?

- RQ3: What is the impact of the community on an open source crowdfunding campaign according to the organizers perspective?

Due to the scarcity of previous literature on the specific topic, we opted for a qualitative research method which enables handling of ambiguous data and paves the way for the subjects' viewpoints. Moreover, it leads to a more in-depth description of characteristics, settings and practices which would otherwise not emerge with quantitative methods. Stemming from the open-ended goal of our research, we conducted semi-structured interviews to collect the necessary empirical data. This technique allows the suggestion of further follow-up questions, formulating a dialogue through the question-answer approach.

The initial goal was to conduct face to face interviews with campaign organizers from Sweden. However, when this was proven neither possible nor sufficient we shifted our attention to the global scene. The interviewees were sought after in major online crowdfunding platforms, i.e. Kickstarter, Indiegogo and Crowd Supply. The primary criteria for selecting campaigns included open source elements to have been developed for the respective products as well as contact information to be available. Altogether, we approached 53 campaign organizers via their email and social media accounts. 20 of them responded. Eventually, 12 interviews were conducted via Skype and 1 via email. The majority (8) of the projects published both software and hardware, 2 of them only software and 1 just the hardware. The rest planned an open source release in the near future. 
Based on the research questions, we compiled an interview guide consisting of 20 interviews, which were slightly modified on the course of time to suit each case better. This was deemed necessary as some questions were not always applicable. For example, some worked alone or had a well-established business prior to the campaign while others worked in teams or did not create a company at all. To ensure participants did not feel distant or estranged, they were introduced to the topic via emails prior to the interview and whenever the connection quality permitted, video calls were made.

\section{RESULTS \& ANALYSIS}

During the interviews, we collected a plethora of data, documenting the experiences, perspectives and actions of 13 campaign organizers. This section shall present some interesting excerpts, present and discuss the collected information as well as correlate them, where applicable, with existing literature.

\subsection{Characteristics}

"I want to be someone who contributes back because I'm rather taking huge advantage of other people's work." - Study participant

The motivation behind organizing a campaign of an open source product can be split into four categories. The first concerns creating inspiration and facilitating innovation through the released source, in exchange for feedback and help. This was also seen in the elements of open communication and shared improvements that constitute the pillars of the hacker culture and techno-meritocracy [11. Moreover, organizers perceived the crowdfunding campaigns as the means to popularize their product, by broadening the awareness around it and its domain. The third perspective that was encountered during this research was that hobbyists (e.g. Makers) often desire or expect products to be open source. Finally, some viewed open source as a gesture of gratitude for the help they had received. This sense of altruism and identification is also encountered in [7].

Next, using a bottom-up approach, such as crowdfunding, makes the campaign perceived as less profit-oriented. Due to the open source nature of the products, the organizers feel like they get more publicity among hobbyists and enthusiasts. Releasing the code makes it easier to reach the crowd and inspires generosity, goodwill and altruistic feelings which translate into financial support for the campaign. Interestingly, keeping supporters in suspense was an explanation for not releasing the source before the campaign. Therefore open source is not just the catalyst that increases engagement and loyalty but also a marketing tool, which is in alignment with the findings of Krishnamurthy 17.

Furthermore, crowdfunding campaigns often appeared to be the sole viable option for commercializing a niche open source product. One of the reasons is that customers who can provide most revenue, such as businesses and public institutions, will not normally take the risk to purchase an unfinished product. 
Secondly, many investors either do not take open source seriously, seeing the product as "less professional" or worse, view open source as a threat. From their perspective, a competitor can utilize the existing source to beat the original developers to the market. Lastly, if a community has already been formulated around a project, crowdfunding is a relatively secure way to gather resources, as it is likely for the community to support it financially. This can be linked to the research of Gerber, Hui and Kou (2012), who argue about the extrinsic motivation of campaign organizers in the pursue to secure funding [3].

"You're using Kickstarter, not only to get the money but also to raise awareness and to start building a community. With open source, the only way it can survive is people using and contributing to it every day, so, it's really gotta be a thriving community." - Study participant

Open source, being a factor that facilitates the formulation of communities, appears to integrate well with crowdfunding campaigns. During the interviews, an organizer mentioned the importance of early community members who, with their ideas and discussions, helped to boost the crowdfunding campaign, especially in the beginning. This importance is in pair with the observations by Lu, Xie, Kong and $\mathrm{Lu}$ (2014) on the relation between the outcome of a campaign and early promotional activities [8]. Moreover, the positive effects of the open source nature are also prevalent after the campaign when the supporters get their hands on the product and start to engage or contribute to it in diverse ways. These factors deem open source highly compatible with crowdfunding.

"We encourage copying. We spread the word and we give all the things you need to create your own project. [..] The only thing we try to work against is the use of our brand name." - Study participant

Having a product open exposes it to the risk of getting cloned and beaten to the market. This can increase competition and damage the profitability. To tackle this, some of the interviewees did not release the source until the end of the campaign. What is more, a proposal for mitigating this risk involved keeping parts of the product closed, such as the hardware. On the other hand, others were not as negative when it comes to cloning. They either embraced it as inevitable and a sign of success or viewed it as a non-threat since the existence of a dedicated community around the product would allow it to be financially viable. Protection against clones can explain the organizer efforts to present themselves as equals among the community and not as a party that simply intends to financially exploit them.

Additionally, it is usually expected for a crowdfunding campaign of an open source product not to offer just software. Particularly, software is expected to come at no cost. More importantly, it became evident that the target audience prefers to pay for hardware or services around the software, rather than just the software itself. This can be interpreted by the fact that the source of the software, once acquired, can be easily transformed into a functional artifact. This 
is not the case with hardware which needs to be fabricated, the necessary components to be acquired at a retail price and assembled. Equivalently, additional software services (e.g. cloud storage or servers) are also hard or costly to set up by individual users, therefore they would be more inclined to purchase them. We documented tendency to support products, someone would have to pay for regardless of whether they were open source or not.

Most organizers initially had no other goal than to test the product's marketability. A formal business perspective surfaced along the way. Their crowdfunding goal became to eventually establish a line of products, reach out to a big market and grow a business. Surprisingly or not, the participants showed no awareness of open source business models. That being said, the model they mainly identified themselves with was that of the support seller [19. The interviewees aligned themselves with the specific model on the grounds of the support that they and their communities offer to each other. In this instance, support is given for free. However, we can perceive the hardware, that typically accompanies the software, as packaging that is shipped to the campaign backers. Therefore, the combination of packaging, distribution and branding feasibly places these cases under the support seller business model.

\subsection{Relationship}

"A community is much like a garden. Fertile soil, plenty of water, plenty of attention and you know, sometimes, as well, it's a bit of luck. It's good weather. But you get out of a community as much as you put in. And only by being incredibly active and giving a community the tools and the resources they need to become engaged, is the only way you can not only build a bigger community but build a more powerful community. One that can really empower themselves." - Study participant

Looking at the relationship between the communities and the organizers, we should first note that we did not discover any norm as to when a dedicated community, around the product, is created. Moreover, previous involvement in open source communities and projects did not play a significant role. This might come as a surprise, as one could assume prior engagement with the community would help the audience identify themselves easier with the organizers.

With Makers being the primary target group, both online and offline communication channels were utilized. The most popular online channels were Twitter, Facebook and mailing lists. Interestingly, some of the organizers maintained separate channels, based on technical skills. The most common offline means were talks on conferences and participation in Maker Faires. Despite many engaging face-to-face with their target group, some participants stressed how these types of activities are time-consuming and ultimately not effective during a campaign.

Furthermore, we saw that involvement with the communities came with mutual communication and a close connection. This involved shared attitudes, reciprocity, selflessness and a sense of goodwill. Many organizers became emotionally tangled with the communities, concerning them as family members rather than 
merely customers and sensing a bond to them regardless of geographical location. Moreover, four elements surfaced, outlining the organizers' role: listening, asking, answering and supporting the communities. Important elements that characterized the communication was selflessness and a positive, proactive attitude. The latter was worded as "being nice" while the campaign organizers mentioned they witnessed a similar stance as a response.

This show of goodwill can be traced in Brabham (2010) who explains how members are enthusiastic and committed to the same cause as the organizers, recognizing themselves as more than just customers 4]. Community members not identifying as mere customers but as organic parts of the project is an attribute also prevalent in the hacker culture ethics. They have the power to influence its direction, help it evolve and are defined by reciprocity and altruism [20].

\subsection{Impact}

"Maker community doesn't like closed sourced products. From a marketing perspective, they are more into open source." - Study participant

To evaluate the impact of the community on a crowdfunding campaign, we examine its effect on the following activities: a) the promotion, b) the received contributions and feedback and c) the business aspects.

As discussed previously, communities can catalyze the reach of a campaign and boost the marketing efforts. A noteworthy factor regarding the promotion of the products was that the majority did not have open source as their primary marketing point. They preferred to focus more on facts, need and usage. Therefore, it is not surprising that some of the interviewees claimed open source had no major effect on their publicity. Furthermore, two of them pointed out mainstream media proved to be unfamiliar with the field or regarded it as unprofessional. On the contrary, the hobbyist community valued open source. Thus, despite open source not being the primary statement of the promotion, it did eventually become relevant since organizers had to be careful not to depict themselves as "too business oriented". A company's intentions to generate revenue can conflict with the open source communities' norms and value, therefore great attention should be paid to keep everyone aligned.

Next, in most occasions software was released after the campaign, therefore limiting the interaction with the community to inquiries regarding the functionality and feature requests. The most significant reasons behind this were to ensure a final bug-free product and avoid being beaten to the market. In the case of products that had released the source before the beginning of the campaign, all but two received a large amount of contribution. It typically consisted of features, usability remarks, code issues and bug reports. Furthermore, a point that was often stressed out, was the importance of listening to feedback. Feedback would come in forms of suggestions, constructive criticism and in some cases negative and questionable comments about the work done on the product. Sorting through it could be time-consuming, but still proved to be vital for a 


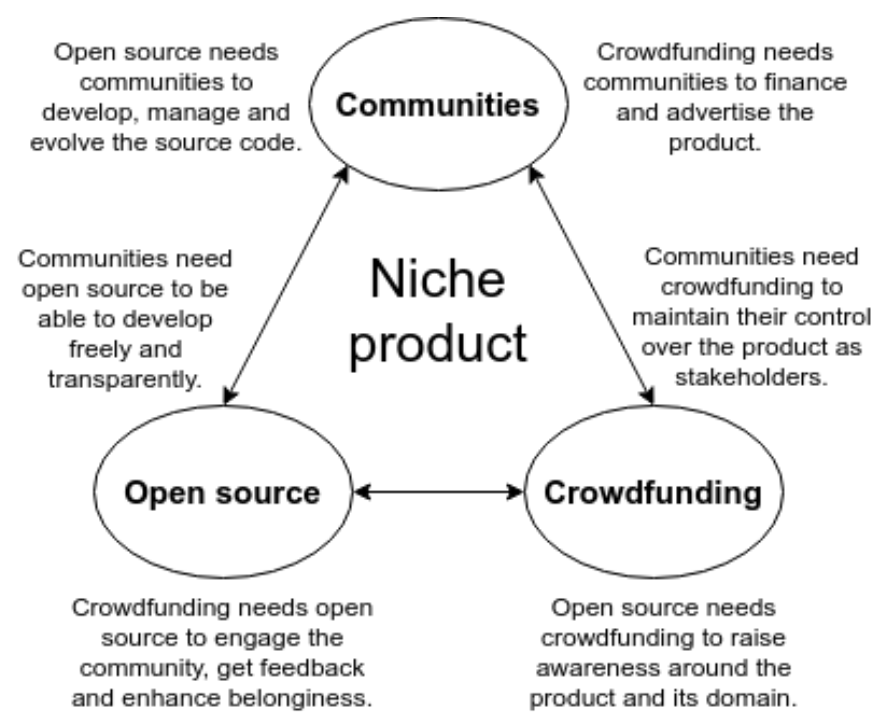

Fig. 1. Dependencies around a niche product

healthy relationship with the community members as well as for the progress of the product.

Nearly half of the campaign organizers aimed to create a line of products utilizing the crowdfunding campaign as the basis for this initiative. The existence of a vibrant and active community that offers feedback, develops and raises awareness can be viewed as fundamental for a long-lasting and successful product ecosystem. Additionally, most of the products involved in this research should be considered as high and low profile nichers targeting a crowd that values open source. This target group expects parts of the product to be open and therefore this translates into going down the open source path, business-wise.

Moreover, crowdfunding is often regarded as the only viable option to finance an open source product. Combining these two points, we come to realize that the impact of the community is not only the deciding factor behind the product being open source but also behind selecting crowdfunding as the means to commercialize it. By taking a step back we can generalize the above observation. Figure1 1 visualizes the interdependent relationships between crowdfunding, communities and open source around niche products. We discover the coupling between these three concepts compels the creators to open source and crowdfund their product, once they attempt to gain the support of a relevant community.

\section{VALIDITY THREATS}

Threats to validity is a typical concern related to open-ended, qualitative studies with semi-structured interviews. This can be, up to a point, attributed to authors having to use creativity, critical thinking, improvisation and therefore 
exhibit a certain degree of subjectivity in order to maintain vivid and interesting discussions [21. We utilized the scheme by Runeson et. al. (2009) [22] to classify the different threats so to employ mitigation tactics against them.

Internal validity is related to risks around the research process which increase the bias. We made an effort to approach a diverse crowd in terms of technology, geographical location and sex. However, the responses we received were primarily by males who were involved in campaigns of products that had both software and hardware elements. Additionally, one interview was conducted via email, instead of a call, which could have inadvertently influenced the collected data.

Furthermore, most of the cases appealed to a rather specific market segment, i.e. Makers and Hackers. This constitutes a serious external validity constraint, thus we do not deem it feasible to generalize our results beyond this class of products. Moreover, participants were selected on a rather ad-hoc basis, having to manually determine whether they developed open source elements as well as the availability of contact details. This leaves room for selection bias. To minimize it we tried to involve a large and diverse sample which also offers us a degree of reproducibility. Lastly, interviews were conducted in English, i.e. not the native tongue of all participants, which could threaten construct validity.

\section{CONCLUSION}

Open source and crowdfunding share a common pillar, communities, which enables their union to be compatible and often fruitful. The first question when contemplating upon open source being crowdfunded is why would someone assume the risk of investing in a product not yet in the market, considering that at some point in time its source will be released. It appears what backers are usually willing to pay for is, difficult to reproduce, hardware that incorporates open source software. Open source and the potential of a developer community around the product safeguard its evolution and user support.

Communities are perceived to play an important role in crowdfunding campaigns. Metaphorically speaking, if sales to high-profile customers allow a product to walk then communities, supporting it through its early stages, allow it to stand up. This importance becomes even more prevailing when open source is involved, as communities are one of the determining factors behind the success or demise of an open source project. Campaign organizers repeatedly emphasized the need to engage as often as possible with the community and place themselves among their customers to increase the sense of belongingness. Moreover, they avoided appearing as business-oriented outsiders who just want to sell a product.

Furthermore, launching a product with low customer applicability targeting communities accustomed to open source, paves the way for the product itself becoming open source and eventually acquiring resources via a crowdfunding campaign. This signifies the magnitude of influence communities have on such products. As future research, we suggest the verification our results through the prism of backers as well as examining the platform characteristics that facilitate crowdfunding of open source projects. 


\section{References}

1. Bagozzi, R., \& Dholakia, U. (2006). Open Source Software User Communities: A Study of Participation in Linux User Groups. Management Science, 52(7), 10991115 .

2. Marketwired. (2015, March 31). Crowdfunding Market Grows 167\% in 2014: Crowdfunding Platforms Raise \$16.2 Billion. Retrieved March 10, 2017 from https://goo.gl/v5QyrG

3. Gerber, E, Hui, J. \& Kou, P. (2012) Crowdfunding: Why People Are Motivated to Post and Fund Projects on Crowdfunding Platforms. Northwestern University, Creative Action Lab, USA.

4. Brabham, D. C. (2010). Moving the Crowd at Threadless: Motivations for Participation in A Crowdsourcing Application. Information, Communication\& Society, 13(8), 1122-1145.

5. Budhathoki, N. R., \& Haythornthwaite, C. (2013). Motivation for Open Collaboration: Crowd and Community Models and the Case of OpenStreetMap. American Behavioral Scientist, 57(5), 548-575.

6. Lakhani, K. R., \& Wolf, R. G. (2005). Why hackers do what they do: Understanding motivation and effort in free/open source software projects. Perspectives on free and open source software, 1, 3-22.

7. Hars, A., \& Ou, S. (2001). Working for Free? Motivations of Participating in Open Source Projects. In System Sciences, 2001. Proceedings of the 34th Annual Hawaii International Conference (pp. 25-39). IEEE.

8. Lu, C. T., Xie, S., Kong, X., \& Yu, P. S. (2014, February). Inferring the Impacts of Social Media on Crowdfunding. In Proceedings of the 7th ACM International Conference on Web Search and Data Mining (pp. 573-582). ACM.

9. Matheus, A. A. R. (2016). The Role of Online Crowdfunding Communities in Funding Cycle Success: Evidence from Kickstarter. Universidade do Porto.

10. Bard, A., Brännström, E. \& Fahlberg, D. (2014). Crowdfunding - En ständig kommunikation. En studie på en communitys relevans runt ett crowdfundat projekt. Institutionen för informatik. Umeå Universitet.

11. Castells, M. (2002). The Internet Galaxy: Reflections on the Internet, Business, and Society. Oxford University Press on Demand.

12. Li, D., Browne, G. J., \& Wetherbe, J. C. (2006). Why Do Internet Users Stick with a Specific Web Site? A Relationship Perspective. International Journal of Electronic Commerce, 10(4), 105-141.

13. Wu, J. J., Chen, Y. H., \& Chung, Y. S. (2010). Trust Factors Influencing Virtual Community Members: A Study of Transaction Communities. Journal of Business Research, 63(9), 1025-1032.

14. Parks, M. R. (2011). Social Network Sites as Virtual Communities. In Papacharissi, Zizi (Ed.) A Networked Self: Identity, Community, and Culture on Social Network Sites (pp.108-109). USA: Taylor \& Francis.

15. Borg, E. (2003). Discourse Community. ELT Journal, 57(4), 398-400.

16. Wasko, M. M., \& Faraj, S. (2005). Why Should I Share? Examining Social Capital and Knowledge Contribution in Electronic Networks of Practice. MIS Quarterly, 35-57.

17. Krishnamurthy, S. (2003). An Analysis of Open Source Business Models. MIT Press.

18. Raymond, E. S. (1999). The magic cauldron.

19. Raymond, E. S. (2001). The Cathedral and the Bazaar: Musings on Linux and Open Source by An Accidental Revolutionary. USA: O'Reilly Media, Inc.

20. Raymond, E. S. (2003). How to become a hacker. Database and Network Journal, $33(2), 8-9$.

21. Maxwell, J. A. (1992). Understanding and validity in qualitative research. Harvard Educational Review, 62(3), 279.

22. Runeson, P., \& Höst, M. (2009). Guidelines for conducting and reporting case study research in software engineering. Empirical software engineering, 14(2), 131. 